\title{
PARASITIC EYE DISEASE IN INDIA AND THE WORLD- A MAJOR REVIEW
}

\author{
Somnath Sarkar1, Tapas Kumar Bhattacharayya², Nivedita Choudhuri3 ${ }^{3}$, Atanu Roy ${ }^{4}$
}

${ }^{1}$ Associate Professor, Department of Ophthalmology, Mata Gujri Memorial Medical College and Lions Seva Kendra Hospital, Bihar. ${ }^{2}$ Associate Professor, Department of Microbiology, Mata Gujri Memorial Medical College and Lions Seva Kendra Hospital, Bihar. ${ }^{3}$ Assistant Professor, Department of Ophthalmology, Mata Gujri Memorial Medical College and Lions Seva Kendra Hospital, Bihar. ${ }^{4}$ Professor and HOD, Department of Microbiology, Mata Gujri Memorial Medical College and Lions Seva Kendra Hospital, Bihar.

\section{ABSTRACT}

\section{BACKGROUND}

Parasites are a group of eukaryotic organisms. They may be free living, may form symbiotic relationships or form parasitic relationships with the hosts. The total number of recognised species of parasites are over 800,000 . They may be unicellular or multicellular. Unicellular parasites include protozoa, while multicellular parasites include helminths and arthropods. The parasites are associated with human beings since the beginning of civilisation. The parasitic diseases are present worldwide including India. In addition to the already prevalent diseases, newer diseases are emerging in the clinical scenario. The systemic diseases like cysticercosis, paragonimiasis, hydatidosis and toxoplasmosis are common. Acquired toxoplasma infections are rising in incidence in immunodeficient individuals. Cestodes, trematodes, tissue flagellates and sporozoa affect human beings commonly including their eyes in different parts of the world. They thus constitute a real health problem globally. The basics of parasitology lie in morphological and pathological studies. This scope has extended further with recent updates in molecular parasitology. The present review discusses the principal ocular parasites found in India and the world with reference to microbiological and pathological aspects and gives outlines of management.

\section{KEYWORDS}

Cysticercosis, Diffuse Unilateral Subacute Neuroretinitis, Hydatidosis, Molecular Pathology, Onchocerciasis, Ophthalmomyiasis, Leishmaniasis, Acanthamoeba Infection, Toxocariasis, Malaria, Toxoplasmosis, Parasitology, Zoonoses.

HOW TO CITE THIS ARTICLE: Sarkar S, Bhattacharayya TK, Choudhuri N, et al. Parasitic eye disease in India and the world- a major review. J. Evolution Med. Dent. Sci. 2017;6(78):5557-5568, DOI: 10.14260/jemds/2017/1207

\section{BACKGROUND}

As early as the time of Hippocrates, parasites were known as agents of human diseases. With the discovery of the simple microscope by Anton Van Leeuwenhoek in 1674, evidence of parasitic diseases were proven. Leeuwenhoek himself drew diagrams of Giardia Lamblia, after seeing the parasite in his own stool through his own microscope.

Parasites are eukaryotic single celled or multicellular microorganisms that grow or multiply on or in host cells and frequently cause disease in man. Ocular parasitology studies parasites that cause human disease and eye complications. ${ }^{1,2}$ Hosts are of two types. The definitive host harbours the adult stage of the parasite or is the host where in the sexual reproduction of the parasites take place. In intermediate hosts, either the larval stages of parasites are found or the asexual reproduction of the parasite takes place. ${ }^{3}$

Many a times, vectors transmit the parasite to the host. The vectors are arthropods. They are of two types- biological and mechanical. The biological vectors provide shelter and also allows multiplication of the parasites, whereas in mechanical vectors, the parasites do not multiply- they are just transmitted to the host.

'Financial or Other Competing Interest': None.

Submission 23-08-2017, Peer Review 17-09-2017,

Acceptance 23-09-2017, Published 28-09-2017.

Corresponding Author:

Dr. Somnath Sarkar,

Satabdi Apartment,

Flat - 4, 333/D, Jessore Road,

Kolkata-700089,

Po+Ps - Lake Town,

West Bengal.

E-mail: som29sarkar@gmail.com

DOI: $10.14260 / j e m d s / 2017 / 1207$

\section{(c) (1) ()요}

There are different routes of entry of the parasites such as ingestion, transcutaneous transmission, insect bite and the haematogenous route. They may be waterborne or there may be simple deposition of eggs. The flies may deposit eggs in the conjunctival sac or orbital wounds and these eggs develop into larvae. This condition is known as myiasis. The larvae may migrate from the paranasal sinuses into the orbit- this condition is known as myiasis interna. Eggs may be deposited in the conjunctival sac and may reach the limbus or sclera to become intraocular, e.g. in thelazia.

The pathology of parasitic diseases are due to mechanical, metabolic and immunological changes brought about by the interaction among the host and the parasites. The principal ocular parasites are discussed below.

\section{Protozoa}

\section{Acanthamoeba}

There are free living amoebae found in fresh water, air and soil. Some species are commensals of oropharynx.

The most important ocular pathogen is Acanthamoeba culbertsoni. The commonest ocular disease is acanthamoeba keratitis. There may be iridocyclitis, scleritis, ocular hypertension and cataract. The organism can cause fatal meningoencephalitis. The organism is found in both trophozoite and cystic forms. Both forms are infective. Both the trophozoite and cystic forms are visible as refractile spots in the confocal microscope. They are also visible in bright field microscope and phase contrast microscopes; 10\% potassium hydroxide mount, Gram's stain and Giemsa stains are also used to identify them. They can be seen under fluorescent microscope with Calcofluor-white stain. ${ }^{4}$ The organism can be cultured in the laboratory under aerobic condition in blood or chocolate agar with overlay of dead E. coli or Klebsiella. The growth appears after overnight incubation and it is detected as areas of clearing of bacterial 
film around the areas seeded with the parasite. Polymerase chain reaction (PCR) detects the parasite rapidly. ${ }^{5}$

Acanthamoeba are transmitted through contaminated warm water in nature and in swimming pools with poor chlorination. They may be inhaled. The most important routes of ocular infections are through contact lens, contact lens storage cases and the contact lens solutions. Trauma or corneal erosion from the contact lens may predispose to keratitis. The patients with HIV infection are more prone to have the disease. ${ }^{6}$ The incubation period may vary between few weeks to few months.

The medical treatment is protracted and frustrating to the patient and the doctor, because the drugs act slowly against the trophozoites. Cysts are very difficult to destroy by drug therapy. The organism is not susceptible to usual antiamoebic drugs. Sometimes they can be treated with antifungals like imidazoles. They are sensitive to aminoglycosides. Some biocides like chlorhexidine and polyhexamethylene biguanide act favourably. Neomycin and Brolene has been used with some success. Cycloplegia is done by atropine sulphate eye drop Penetrating Keratoplasty and the use of tissue adhesives along with bandage contact lenses have improved the visual outcome in the patients.

\section{Toxoplasma}

The genus Toxoplasma has only one species, Toxoplasma gondii. The organism is present in soil and water. This is an obligate intracellular coccidian parasite. This is now recognised as the most common protozoan parasite globally. This has a wide range of hosts spread over two hundred species including mammals, birds and reptiles. The domestic cat is its definitive host, where the sexual sporogony takes place. About $50 \%$ of the population in developed countries have serological and clinical evidences of the disease, whereas the prevalence rate is much higher in developing countries.

The organism can produce disease at any age in both sexes. It can either be congenital or acquired. There may be reactivation or recrudescence. Primary infection with the parasite may be asymptomatic, acute or chronic. In chronic infection tissue, cysts are produced within muscles and other tissues. Human infection is a dead end for the parasite. Human infection may occur in different ways such as eating uncooked or undercooked infected meat containing tissue cysts; ingestion of mature oocysts through food, water and contaminated fingers from cat faeces. They may pass from mother to foetus through placenta. There are some evidences where the organism has been transmitted through blood transfusion or transplantation of organs.

The ocular infection is either congenital or secondary to systemic diseases. Ocular disease is milder than systemic disease. The immunocompromised persons have a higher incidence of fatal outcome. Infectious retinitis and posterior uveitis may occur.7,8 "Searchlight in fog" appearance is characteristic clinically.

Laboratory diagnosis includes staining and microscopy, isolation of the organism and serological tests. Giemsa stained impression smear may occasionally show the characteristic trophozoites. Tissue section may reveal the cystic forms. Isolation may be done by injecting body fluids, ground tissues into cell cultures and intraperitoneally in immunocompromised mice. The peritoneal fluids and spleen smears from the mice may show the trophozoite after $7-10$ days. Serial blood passages may often be required for isolation. The most common method of laboratory identification of the disease is done by serological tests detecting antibodies. These include the Sabin-Feldman dye test, indirect immunofluorescence, haemagglutination, complement fixation test and ELISA. The standard test employed nowadays is ELISA for IgM and IgG antibodies. Detection of only IgM indicates recent infection. Low IgM titre with high IgG titre suggests infection in the recent past; no IgM but high IgG goes with past infection. Serial ELISA tests provide better information than one isolated test. Immunoproteomic technology has provided a useful tool for its diagnosis. ${ }^{9}$

The organism is sensitive to many antimicrobials such as sulphonamides, antibiotics and antimalarial drugs. Combined therapy with pyrimethamine and sulphonamides or cotrimoxazole (Daraprim: pyrimethamine and sulphadiazine) may result in a clinical cure. However, the parasite may not be eliminated. Spiramycin and Clindamycin have also been used. The treatment is effective only against trophozoites. No drug is available against the cystic forms. Tissue cysts present in untouched retinal tissue may develop infection later, ${ }^{10}$ particularly after injudicious use of corticosteroids. ${ }^{11}$ Bradyzoites have showed the capacity of growth later on. ${ }^{12}$

\section{Microsporidia}

Microsporidia are classified under the phylum Microspora. They are obligate intracellular protozoa. They have been recognised as animal parasites for long, but their role as human pathogens has been revealed only in the mid-1980's with the spread of HIV infection. Some nine genera and thirteen species of Microspora are associated with human disease, particularly in immunocompromised hosts. They can cause a wide range of illness from diarrhoea to involvement of central nervous system, eyes, viscera and disseminated disease. The most common species that can cause human disease are Enterocytozoon bieneusi and Encephalitozoon species. Others include Brachiola, Nosema, Pleistophora and Vittaforma species. The main organisms affecting ocular tissues are Encephalitozoon and Nosema.

The exact mode of transmission is not well documented. It may be by ingestion or droplet infection. The ocular infection spreads by fingers contaminated by urine and stool. Other predisposing factors are trauma and close proximity with infected animals.

The ocular diseases are punctate epithelial keratitis, keratoconjunctivitis and stromal keratitis.

The diagnosis is by demonstration of the organisms in biopsy specimens.

Conjunctival scrapings may show the organism. Fluorescent microscopy, confocal microscopy and electron microscopy help in identification. Serology and culture are not useful.

No effective therapy is known for systemic infection. There are some drugs like metronidazole, thiabendazole, trimethoprim and albendazole which are used for systemic disease. Local ocular treatment includes application of fumagilline $70 \mu \mathrm{g} / \mathrm{mL}^{13}$ or propamidine isethionate drops. 


\section{Pneumocystis carinii}

The taxonomic status of P. carinii has been uncertain. It is generally considered as a sporozoan parasite. However, analysis of its mitochondrial and chromosomal genes indicate that it has more intimate relation with fungi. ${ }^{14}$ It occurs in two forms, trophozoite and cyst. Some trophozoites become encysted and produce eight daughter trophozoites within the cyst (Intracystic bodies or sporozoites).

Natural infection with P. carinii (now better known as P. jirovecii) occurs in many species of animals. Human infection may come from infected dogs or other domestic animals, but this is considered much less important than human to human spread. It is transmitted through droplets, as it is a commensal of the lungs.

The only known ocular disease is choroiditis. This occurs by haematogenous spread from respiratory infection. The most susceptible persons are immunocompromised persons, especially those having HIV infection, ${ }^{15}$ premature babies and neonates.

The diagnosis is confirmed by demonstration of trophozoites in the specimen. The histopathological sections are stained by Gomori's methenamine silver stain, toluidine blue, PAS and Calcofluor white. The organism is not stained by haematoxylin-eosin stain. The sporozoites are stained by Wright's and Giemsa's stain. Immunofluorescence using monoclonal antibodies and PCR are very sensitive and specific.

The treatment is done by trimethoprimsulphamethoxazole and pentamidine isethionate. However, recurrence is common. Dapsone is also used, but has a higher incidence of side effects. ${ }^{16}$

\section{Leishmania}

This is an infection caused by protozoa belonging to the genus Leishmania. The two species causing infection are L. donovani and L. tropica. In India, the disease is mainly prevalent in Bihar, Jharkhand, parts of West Bengal and Odisha. The disease is spread by the sand flies of the genus Phlebotomus and Lutzomyia. ${ }^{17}$ It causes fever (Kala-azar) with darkening of skin, reduction in immunity, occurrence of opportunistic infections, occurrence of tuberculosis in many patients and ultimately wasting, emaciation and death. It can occur as co-infection in HIV-affected individuals. In such patients, the eye may be affected. These patients often show relapse after treatment. ${ }^{18,19}$

Ocular affection may be sight threatening. In the skin, the lesions are swelling, papule, plaque, nodule or ulcer. Ptosis, lagophthalmos, ectropion of lower lid, lacrimal discharge, ulcerative blepharitis, blepharoconjunctivitis, nodular episcleritis, scleromalacia, ulcerative interstitial keratitis, anterior uveitis and macular haemorrhage all can occur. Biswas et $\mathrm{al}^{20}$ reported a patient of spontaneous resolution of bilateral macular haemorrhage in kala-azar infection. On histopathological examination, numerous intracellular and extracellular Leishman-Donovan bodies are also seen.

Treatment is with sodium antimony gluconate or pentamidine preparations. Ocular affections may be treated by steroids or lubricants.

\section{Malaria}

This disease is caused by the genus Plasmodium, having four different species: vivax, ovale, malariae and falciparum. It is transmitted by the bite of female Anopheles mosquito. The common species are P. vivax and P. falciparum. ${ }^{1}$

The ocular complications occur in about 10 to 20 percent of patients. ${ }^{21}$ The commonest complications in the acute phase are conjunctival hyperaemia and subconjunctival haemorrhage. There may be yellowish discoloration of the conjunctiva. 22

Retinal haemorrhage, transient ocular nerve palsies, orbital oedema, choroiditis, retinal embolism, papilloedema and paralysis of pupillary light reflex and accommodation can occur. Herpetic keratitis can develop after malarial infection due to immune dysfunction. ${ }^{23}$

P. falciparum can be sequestered in the capillary and venule microvasculature. It can occlude vascular lumen by cytoadherence and rosette formation. The mechanism of cytoadherence is by development of ligand associated knobs on the surface of infected erythrocytic membranes. ${ }^{21,23}$ This leads to thrombosis, occlusion and haemorrhage.

The malarial pigment presents microscopically as black to dark brown granules. The presence of haemozoin is regarded as diagnostic of malaria. ${ }^{21}$ The presence of ocular lesions in malaria heralds a bad prognosis. This is more so in patients of cerebral malaria. ${ }^{23,24}$ Thus patients having large unexplained retinal haemorrhage, especially in India should be thoroughly investigated for malaria.

The diagnosis of malaria may be established by dual malarial antigen test in addition to microscopic demonstration and diagnosis of malarial parasite species in thick blood films stained by Leishman's stain.

The disease is treated by chloroquine phosphate. However, P. falciparum is now often treated with artemether preparations derived from the drug artemisinin obtained from the Chinese herb Artemisia annua (Qing hao su). Quinine obtained from cinchona bark can be used, but has significant side effects. Daraprim may also be used in the treatment. Fluid therapy, corticosteriods and ventilatory support may be given in severely ill patients, who present with Adult Respiratory Distress Syndrome (ARDS).

\section{Giardia lamblia}

This causes giardiasis. Acute cases present with diarrhoea, abdominal pain, bloating, belching, flatulence, nausea and vomiting. In the chronic stage malabsorption, steatorrhoea, multiple recurrent attacks of diarrhoea and weight loss may occur. This disease can cause growth impairment in children.

The reports of ocular infestations are usually secondary to hypersensitivity to Giardia antigen. This is because the parasite has never been found in extraintestinal tissue. The ocular changes include anterior uveitis, choroiditis and retinal pigment changes. ${ }^{25}$ The disease is treated by metronidazole therapy or quinacrine therapy.

\section{NEMATODES}

The phylum Nematoda comes under kingdom Metazoa. They are also called roundworms. They are of three types:

a. Intestinal species.

b. Intestinal-tissue species.

c. Blood-tissue species.

The nematodes which can cause ocular disease include Toxocara, Thelazia, Baylisascaris, Trichinella, Onchocerca volvulus, Gnathostoma spinigerum, dirofilaria, Ascaris 
lumbricoides, Loa, Dracunculus medinensis and Wuchereria bancrofti.

The nematodes are elongated, cylindrical and unsegmented worms with tapering ends. They are bilaterally symmetrical. The body is covered with a tough cuticle. The body cavity is a pseudocoele in which all the viscera are suspended. They have a complete digestive system. They have a simple excretory and nervous system. The sexes are separate. Most nematodes are free living and are present in soil and water. The largest number of helminthic parasites of human beings belong to the class of nematodes.

The commonest route of infection is the faeco-oral route. Some larvae can penetrate the intact skin. Some can pass through inhalational route, while others can be transmitted by the bite of blood sucking vectors.

\section{Toxocara}

This is the most commonly reported nematode to cause ocular diseases. It is an ascarid of dogs (T. canis) and cats (T. cati). The role of $\mathrm{T}$. cati as a pathogen in human beings is doubtful. Ocular toxocariasis is caused mainly by T. canis. ${ }^{26} \mathrm{~T}$. canis infection occurs mostly in children. Systemic involvement has been reported globally. Ocular disease is quite common.

The infection is caused by ingestion of ova in contaminated food or ingestion of larva from infected meat. The dogs and cats are the definitive hosts. Human beings are considered accidental hosts in whom the nematode develops to third stage larva only. In humans, the third stage larvae in the gut penetrates the mucosa of the intestine and are carried through blood to different organs. They encyst and form granulomas. They cause cutaneous and visceral larva migrans (VLM). They cause ophthalmic larva migrans (OLM).

The systemic infection is difficult to diagnose. A high index of suspicion is required and the diagnosis is mainly one of occlusion. Ocular larva migrans and visceral larva migrans do not coexist. OLM is seen in older children in whom there may be a clinical history of playing with dogs and cats as pet or a history of geophagy (pica) or a history of visceral larva migrans (VLM) a few years ago. There is marked eosinophilia. In ELISA test for toxocara, a titre of 1:8 is suggestive of OLM. Antibody titre in OLM is higher in aqueous than in serum. Splendore-Hoeppli phenomenon also occurs. ${ }^{27}$

Ocular toxocariasis should be differentiated from other causes of white reflex in the pupillary area by indirect ophthalmoscopy, ultrasonography, x-ray, CT scan and MRI. The other causes of white pupillary reflex include congenital cataract, retinoblastoma, Coats' disease and endophthalmitis. Unilateral neuroretinitis with subsequent motile retinal lesion denotes ocular toxocariasis. ${ }^{28}$

Systemic toxocariasis is treated with oral albendazole and thiabendazole. Eye complications are treated with steroids, cycloplegics and by laser therapy to the retina.

\section{Thelazia}

This is a small thin nematode. The life cycle and mode of transmission is not well documented. Ocular manifestations occur by two common species: $\mathrm{T}$. californiensis and $\mathrm{T}$. callipaeda. The adult worm resides in the conjunctival sac, lacrimal sac and lacrimal duct of many animals and birds. The definitive hosts are suspected to be dogs, cats and horses. The intermediate host is the fly of the genera Musca and Fannia.
Human beings are accidental hosts. The flies lay eggs in the conjunctival sac, where they develop into adult worms. The adult worms are seen to move about in the conjunctival sac or subconjunctival space and they may cause pain and watery conjunctivitis. ${ }^{29}$ They may penetrate the cornea or sclera and may become intraocular. Intraocular worms are seen moving freely in the aqueous. They must be differentiated from $\mathrm{W}$. bancrofti, Dirofilaria and Brugia malayi. The death of the worm causes severe hypersensitivity reaction. Hence, it is better to remove the live worm from the aqueous then to use antihelminthic medicines.

\section{Dirofilariasis}

This disease is caused by Dirofilaria immitis or the dog heartworm. The parasite infests the heart and the large blood vessels in dogs. It is found in tropical, subtropical and warm temperate regions of the world. The dog is the definitive host and the mosquito is the intermediate host. It is transmitted to human beings by mosquitoes. ${ }^{30}$ It affects the periorbital space, ${ }^{31}$ subconjunctival space and anterior chamber. ${ }^{32}$ It may mimic a lacrimal sac mucocoele ${ }^{33}$ or even a lid tumour. ${ }^{29}$ It can cause choroiditis ${ }^{34}$ and vitritis. ${ }^{35}$ When human beings are accidentally infected, the larvae do not complete their lifecycle but lodge and obstruct the pulmonary arterioles. Thus, lung affection occurs and timely detection prevents major complications. The microfilaria do not circulate in human blood. A biopsy is diagnostic in the case of a lacrimal sac mucocoele. ${ }^{33}$ Multifocal choroiditis may be caused by it. Retinal pigment epithelial tracts are suggestive of parasitic infection. ${ }^{29}$ D. repens infection has been reported from India. It can cause painful erythematous subcutaneous nodules in and around the eyes. ${ }^{36}$ The laboratory confirmation of dirofilariasis is by identifying the worms on biopsies of the subcutaneous nodules. ${ }^{36}$

\section{Trichinella}

This is one of the smallest nematodes infecting humans. In Asia, trichinosis (trichinella infection) has been reported from Malaysia, Vietnam, Thailand, China and Syria. In India, the first case of human trichinosis has been reported from Punjab in 1996.

The infective form is the encysted larvae found in the muscles of pigs and other animals. The transmission occurs via uncooked or undercooked infected meat. There is excystation in the stomach, duodenum and jejunum. The larvae immediately penetrate the mucosa, moult four times and develop into adult by the second day of infection. The female worm is viviparous. The fertilised females release motile larvae by the sixth day of infection. The larvae enter the intestinal lymphatics or mesenteric venules and are transported to different parts of the body through circulation. After deposition in the muscles, development occurs for the next three to four weeks. The larvae then become encysted. These larvae remain infective inside the cyst for many years. This is the dead end of the parasite. ${ }^{37}$

In the eyes, the larvae invade the orbicularis oculi muscles and can cause periorbital oedema and proptosis. In rare instances it causes retinal haemorrhage, optic neuritis and conjunctivitis. Pain and photophobia may occur. ${ }^{38}$

There is eosinophilia. Serological tests for T. spiralis are most useful. These include ELISA, complement fixation test, indirect haemagglutination test and immunofluorescence 
test. The most confirmatory test is demonstration of encysted larvae in striated muscles by histopathology. ${ }^{37} \mathrm{X}$-rays may reveal calcified encysted larvae. CT scan, MRI and USG all may be helpful.

The commonly used oral drugs are thiabendazole and metronidazole. The drugs are not effective against the encysted forms. ${ }^{37}$ Analgesics may be given for pain. For severe myositis and myocarditis, glucocorticoids may be given. ${ }^{37}$ Thiabendazole is associated with more side effects like nausea, rash, mental changes and Stevens-Johnson syndrome in children. ${ }^{39}$

\section{Baylisascaris}

Baylisascaris procyonis is a roundworm of raccoon. The human beings are accidental hosts. The disease occurs by ingestion of food contaminated by eggs.

The nematode reaches the eyes and brain by the blood stream. They cause visceral larva migrans. In the eyes the live organism can be seen moving in the retina causing retinitis, diffuse unilateral subacute neuroretinitis (DUSN), ${ }^{40,41}$ optic neuritis and retinal vasculitis. DUSN is also known as unilateral wipe out syndrome, causing slow but severe visual loss.

The presence of motile nematode in the retina confirms the diagnosis. They are best visualised by Goldmann fundus contact lens. ELISA testing is of some importance. Anthelmintics like thiabendazole ${ }^{42}$ have limited success in the management. The best management is the destruction of nematode by laser. ${ }^{43}$ Ivermectin or diethylcarbamazine may be tried, probably with moderate success. ${ }^{42}$

\section{Onchocerca volvulus}

The disease caused is known as onchocerciasis. As it is associated with black flies along the banks of fast flowing streams, the condition is also called "river blindness." The filarial worm is most common in parts of tropical Africa and Central America. Currently, 17.7 million are infected with 0. volvulus. Of these, 2,70,000 are blind and 500,000 have severe visual impairment. However, air currents may displace flies into deeper areas where infestation may occur.

The adult worms are located in the subcutaneous tissue. Adult worms are white, transparent with transverse striations on the cuticle. The average lifespan of the female worms is 8 years, but they can live up to 15 years.

The microfilariae are unsheathed. The column of nuclei do not extend up to the tail tip. They are non-periodic. Microfilariae are usually present in subcutaneous tissue and rarely in blood, urine or sputum.

0 . volvulus has two hosts: man, and the day biting female black fly Simulium. Man is the definitive host, while the fly is the intermediate host. The human beings get infected by the bite of female black flies, which have the infective third stage larvae in their mouth parts. The larvae enter into the skin through the bite wound and migrate to the subcutaneous tissues, where they moult twice and mature into adult male and female worms. The females release actively motile unsheathed microfilariae, which migrate through the whole body for about one year before setting in a nodule.

The microfilariae may remain localised to subcutaneous tissue or may migrate to other tissues, notably the eye, causing corneal and retinal lesions that lead to blindness. Ocular lesions are a result of the host immune response to the microfilariae. Ocular changes include punctate keratitis, sclerosing keratitis, anterior uveitis with secondary cataract and glaucoma, chorioretinitis and papillitis. Visual field may be severely constricted. Ultimately blindness supervenes.

On histopathological examination, the areas around dead microfilariae are infiltrated with eosinophils and have oedema. ${ }^{44,45}$ There is a predominance of CD8+ suppressor cells and increased messenger RNA. ${ }^{46}$ Autoantibodies against retinal S-antigen, inner retinal and retinal photoreceptors are found in ocular fluids. This suggests autoimmunity as one of the mechanisms in the pathogenesis. ${ }^{47}$

A rare case, where the worm was extracted from the eye, was reported from India 48 with posterior segment involvement. The visual prognosis is worse.

The diagnosis depends on the demonstration of microfilariae in the anterior chamber of the eye on slit lamp examination. The adult worms can be demonstrated inside the excised nodule. There is marked eosinophilia. The microfilariae are absent in peripheral smear.

The disease can be prevented by vector control and by chemoprophylaxis with ivermectin. The curative therapy includes ivermectin and diethylcarbamazine (DEC).

\section{Gnathostoma spinigerum}

It causes the disease called gnathostomiasis. The disease is caused by the third stage larva of the genus Gnathostoma. This parasitic infestation occurs in the East Asian and far East Asian countries including India.

This parasitic infestation can occur in human beings after intake of raw flesh of the definitive hosts like cat, dog and other wild animals. The commonest species infecting man is Gnathostoma spinigerum. The disease may have cutaneous, visceral and ocular manifestations. It has two intermediate hosts. Cyclops is the first intermediate host, while freshwater fish, frog or snake are the second intermediate hosts. Man may thus also be infected by ingesting Cyclops with drinking water. In man, the parasite remains viable without further development.

Ocular disease occurs due to toxins as well as larval migration. 49 The commonest ocular feature is anterior uveitis. ${ }^{50,51,52}$ There are other ocular features like lid swelling, iris atrophy, iris holes, intraocular haemorrhage, glaucoma, retinal scars and retinal detachment.

The use of scanning electron microscopy improves the staging of the disease as well as the ability to distinguish between different species. 53,54

The treatment includes cycloplegic and NSAID drops and corticosteroids for the uveitis. Pars plana vitrectomy with endolaser and use of silicone oil or sulphur hexafluoride gas may be required in cases of intravitreal involvement with retinal detachment (tractional retinal detachment). If the posterior segment is involved, visual prognosis is uncertain.

\section{Ascaris lumbricoides}

This is a helminthic infection caused by the largest of the human helminths, Ascaris lumbricoides. ${ }^{55}$ The adult worm lives in the jejunum. Human beings acquire infection by ingestion of embryonated eggs. This usually happens if his hands are in contact with soil and are not washed properly before taking food. The larva produces an enzyme ascarase, which is very irritant and may cause allergic manifestations. ${ }^{56}$ 
The body fluid is toxic on absorption and may damage tissues.

The eye may be involved by migration of larva via blood or lymph. It can cause conjunctivitis, uveitis, secondary glaucoma, recurrent vitreous haemorrhage, choroiditis, periphlebitis and papilloedema. ${ }^{57}$ The parasite has been found in the lacrimal passages. ${ }^{58}$ Pars plana vitrectomy may be diagnostic of intraocular infection. 55,58

The disease is treated by oral albendazole and thiabendazole. Uveitis is treated with corticosteroids and cycloplegic drops. Intraocular disease is treated by vitrectomy.

\section{Loa loa}

The disease caused is called loiasis. It occurs in endemic form in the rain forests of Africa (central and western Africa). About 15 million people are infected with the disease. The adult worms live in the subcutaneous connective tissue. Ocular involvement is mostly in the subconjunctival tissue of the eye. The cornea is usually not involved. Thus, the parasite is known as the eye worm.

The sheathed microfilaria are found in the blood. They resemble the microfilaria of Wuchereria bancrofti except that the nuclei extend completely to the tip of the tail. It has a diurnal periodicity; most microfilariae are present in blood at about midday.

The adult worms are thin and whitish. The sexes are separate. They move freely in the subcutaneous tissue and lymphatics. The pre-patent period in human beings is about six months and the adult worm may live seventeen years or more.

L. loa requires two hosts in its life cycle- human beings and the day biting female mangrove flies (Chrysops dimidiata and Chrysops silacea). ${ }^{59}$ Human beings acquire infection by the bite of these flies. From the bite wound, the larvae enter the subcutaneous tissue, moult and develop into adult worms. The development takes about 6 to 12 months. The female worms release microfilariae, which circulate in the peripheral blood during day time. In other times, they reside in the lungs. The sheathed microfilariae are ingested by the female flies during their blood meal. The sheaths are cast off. The microfilariae penetrate the stomach wall and reach the thoracic muscles where they develop into infective larvae. In the female flies, the duration of development is about ten days. The infective forms migrate to the mouth parts of the Chrysops for the next transmission to occur.

\section{The Clinical Features Consist of}

a. Calabar swellings or fugitive swellings, which are painless oedematous swellings under the subcutaneous tissue.

b. Subconjunctival migration of the filarial worm.

The presence of the worm in the subconjunctival space causes itching, foreign body sensation and mild conjunctival hyperaemia. 60 The worms can be seen to move under the conjunctiva. Eosinophilia is present.

The dead worms can cause nodule formation in the conjunctiva as well as acute periorbital angioedema. The tolerance to the worm, when it travels to the anterior chamber, is satisfactory. When the worm dies, extensive iridocyclitis occurs. The aqueous humour becomes cloudy, the vitreous becomes opaque and the intraocular pressure is raised.61 Some cases may show posterior segment involvement. The lesions in the posterior segment include retinal detachment, retinal neovascularisation, subretinal exudates and vitreous haemorrhage. Multiple yellowish exudates throughout the retina and occluded retinal arterioles have been recorded. 62

Systemic manifestation like arthritis, lymphangitis, peripheral neuropathy, encephalopathy, nephropathy and cardiomyopathy have been found.

The laboratory diagnosis is made by demonstration of microfilariae in the peripheral smear during the day. The blood is preferably collected between $10 \mathrm{AM}$ to $2 \mathrm{PM}$. The infection can also be diagnosed by extraction of the worm from the subconjunctival space.

Loiasis is treated by the surgical removal of the adult worms. The most favourable time is when the worms are passing through the conjunctiva. Diethylcarbamazine is the drug of choice. It kills both the adult worms as well as the microfilariae. The dose is $2-6 \mathrm{mg} / \mathrm{kg}$ body weight daily for two to four weeks. Other drugs which are used include albendazole and ivermectin. Antihistaminics, antipyretics and corticosteroids may be used according to the clinical situation.

The prophylaxis consists of vector control, use of repellents on the exposed skin and detection and treatment of infected persons with diethylcarbamazine.

\section{Dracunculus medinensis}

The common name is Guinea worm. It occurs in Africa and the Indian Subcontinent. It has now been eradicated from India.

The adult females measure upto one metre in length and inhabit the subcutaneous tissues of the legs, arms and back. The human beings are the definitive hosts, while the cyclops are the intermediate hosts.

The infecting agent is the larva. The female hatches thousands of motile first stage larvae into the water. They are taken up by the Cyclops, reach mid intestine and penetrate through the soft wall to the body cavity. They moult twice and become infective.

The human beings get infected after drinking water is contaminated with Cyclops. On reaching the stomach, Cyclops are digested by the gastric juice and liberate the larvae. The larvae penetrate the wall of the gut and reach mainly the retroperitoneal region. They mature into male and female adults. The male fertilises the female and dies. The females migrate to the subcutaneous tissue of mainly the hands and feet, where it releases toxic substances resulting in a blister and then ulcer formation.

The ocular involvements are few and uncommon. The eyes are not directly involved. The worm is lodged under the skin of eyelid or in the orbit, forming larvae discharging sinuses. There may be proptosis. The dead worm may be calcified in the retrobulbar space. There may be periorbital odema. ${ }^{63}$ On x-ray, the calcified worm may be detected accidentally. ${ }^{63}$

The drinking of filtered or boiled water in endemic regions reduces the chances of infection. There is no definitive chemotherapy. Thiabendazole, albendazole and 
metronidazole have been reported to hasten the expulsion of the worms. They also act as anti-inflammatory agents.

\section{Wuchereria bancrofti}

This is the most widely distributed human filarial parasite. ${ }^{64}$ It is found in India, China, far East, major portions of Africa, South America and Central America. It causes bancroftian filariasis or lymphatic filariasis. ${ }^{65}$

The human beings are the definitive hosts, while mosquitoes of the genera Culex, Aedes and Anopheles are the intermediate hosts. The infective form for humans is the third stage larva. They are deposited on the skin of human beings near the site of mosquito bite. The larvae penetrate through the puncture wound to reach the subcutaneous tissue. From there they go to the draining lymph nodes and develop into adults in 3 - 15 months. The male fertilises the female worms, which gives rise to sheathed microfilariae. These microfilariae reach peripheral blood during $10 \mathrm{PM}$ to $4 \mathrm{AM}$, from where they are again taken up by the mosquito during blood meal, thus completing the cycle.

The adult worms damage the lymphatic to a good extent. On the death of the worms, there is enhanced granulomatous reaction and fibrosis. ${ }^{65}$ Thus, there is chronic lymph-oedema with elephantiasis. Secondary bacterial infection may occur. The eye is rarely involved in filariasis. It can cause oedema of the eyelids and rarely panuveitis. ${ }^{64}$

The definitive diagnosis is made by the demonstration of the parasite (microfilariae) in peripheral blood. Microfilariae are more in capillary blood and hence blood from ear lobes are preferred.65 Other tests are passive haemagglutination test, fluorescent antibody test and ELISA test.

The treatment consists of DEC therapy. A dose of 6 $\mathrm{mg} / \mathrm{kg} /$ day for two to three weeks clears the microfilariae, but adult parasites may survive. However, there is some effect on the adult worm and hence prolonged low-dose DEC may lead to cure. 65

\section{Cestodes}

They are segmented, dorsoventrally compressed and tape like in appearance. Hence, they are known as tape worms. Their size varies from few millimetres to several metres in length. Adult cestodes live attached to the mucosa in the small gut and absorb food from the host.

Cestodes have three parts- scolex (head), neck and strobila (body). The scolex consists of suckers, grooves or hooks to attach to the surface mucosa. The neck is very short. Strobila is composed of a chain of proglottids or segments. The newly formed segments are proximal and they push the mature segment distally. Each mature segment contains full set of male and female genital organs. Therefore, each individual worm is a hermaphrodite.

In pseudophyllidean cestodes the egg is ovoid, operculated and does not contain any embryo when first laid. In cyclophyllidean cestodes, the egg is operculated and has two coverings. The egg when first laid contains six hooklets, hence called hexacanth embryo.

Cestodes complete their life cycle in two different hosts. However, in Hymenolepis nana, there is only a single host. In most of the human infections, man is the definitive host. An important exception is the dog tapeworm, Echinococcus granulosus, for which dog is the definitive host and man is the intermediate host. For pork tapeworm Taenia solium, man is usually the definitive host. Sometimes, man acts as the intermediate host and harbours the larval form of this parasite. The intermediate hosts are mammals, fish or arthropods. In many cestodes only one intermediate host is needed, but in Diphyllobothrium latum, two intermediate hosts are required.

The cestodes that have ocular involvement include Taenia solium, Echinococcus granulosus, Taenia multiceps and Spirometra.

\section{Taenia solium}

This causes the disease cysticercosis. The cestode resides in the human small gut. The head (Scolex) is attached to the gut wall and grows a large number of bisexual segments called proglottids. These proglottids and free ova are discharged through faeces. Depending on proper temperature and moisture, they can remain alive in the environment for weeks to months.

They are ingested by the intermediate hosts like man, pig, cattle and fish. They hatch in the intestine, penetrate the mucosa and disseminate throughout the body, eventually forming cysts (Cysticerci). These cycticerci give rise to new scolices.

The cysts remain dormant until ingested by a carnivore and then grow info sexually mature worms in the jejunum of these animals.

Taenia solium causes cysticercosis in man and it has ocular significance. They can reach the lymphatic and blood circulation by auto-infection via the ano-oral route or by eating undercooked vegetables, fertilised by nightsoil.

The asexual juvenile form of T. solium (called cysticercus cellulosae) forms cysts in the conjunctiva, anterior chamber in the orbit, under the retina and most commonly in the vitreous humour. ${ }^{66}$ Uveitis may occur. ${ }^{67}$

The treatment is by excision including pars plana vitrectomy and orbitotomy. Albendazole $15 \mathrm{mg} / \mathrm{kg} /$ day is given in divided doses for eight days. ${ }^{68}$ Alternatively, praziquantel $50 \mathrm{mg} / \mathrm{kg} /$ day is given in three doses per day for fifteen days. Prednisolone tab $40 \mathrm{mg}-60 \mathrm{mg}$ per day can be given orally to suppress inflammation. Metrifonate 75 $\mathrm{mg} / \mathrm{kg} /$ day for five days repeated at two week intervals is effective in ocular disease. The patient should undergo deworming also.

\section{Echinococcus granulosus}

The adult tapeworm of E. granulosus lives in the gut of dogs, cats or wolves. The life cycle is similar to that of Taenia. The intermediate hosts are usually sheep, pigs, buffalo, camel and deer. Man may be accidentally infected. The cysts are called hydatid cysts and are located in the liver or lungs. Other organs which are involved are the central nervous system, heart, kidney and spleen. The larva penetrates the eye rarely. The cysts can be found in the eyelids or the orbit.69 Exophthalmos may occur Ultrasonography and CT scan aids the diagnosis. ${ }^{70}$

The treatment is surgical removal of the cyst, which may require orbitotomy. Large cysts should not be removed entirely to avoid accidental rupture and release of viable larva into the tissues. The cyst wall is punctured and the contents are aspirated out. Toxic substances are injected into the cyst and left for five minutes followed by aspiration. The cyst wall is then excised. 
Mebendazole $50 \mathrm{mg} / \mathrm{kg} /$ day divided into 3 doses is given for one month. This is repeated at 3 months and 6 months. Albendazole $800 \mathrm{mg} /$ day in four doses for twenty eight days, repeated twice at two weekly intervals after the first dose gives better results.

\section{Spirometra}

This causes the disease sparganosis. The definitive host is a carnivore. The first stage larva infect tiny crustaceans. Subsequent larvae are called spargana. They infect amphibians and reptiles. Man is infected by drinking contaminated water containing Cyclops and consuming raw frogs, mammals, fish or snakes. Infection may also result from the application of amphibian or reptilian flesh as poultices to the skin or eyes.

In the eyes, painful itchy lid oedema occurs with epiphora. There may be proptosis. The disease is prevalent in China and South-East Asia, but is also seen in parts of India. Subconjunctival larva migrans may occur. ${ }^{71}$

The diagnosis is usually established by examining the surgically removed larva. It is white and tape like with the body being transversely wrinkled with a longitudinal groove. ${ }^{72}$ Surgical removal is the treatment of choice. Intravenous neoarsphenamine may be given.

\section{Taenia multiceps}

This parasite is also called Taenia multiceps. It is a less prevalent dog tapeworm. The larval form resembles both cysticercus and hydatid cyst to some extent. It differs from cysticercosis, in that it contains multiple scolices. It does not contain brood capsule or daughter cysts like hydatid cyst. It is longer than both cysticercus or Echinococcus. It is prevalent in Africa, Europe and USA mainly. ${ }^{73}$

The larval form is coenurus. The dogs or other canines are the definitive hosts. The sheep and other herbivores are intermediate hosts. Human beings are accidental intermediate hosts. The life cycle resembles that of cysticercus.

The ocular manifestations are similar to cysticercosis, but less frequent. They include subconjunctival cysts and cysts in the anterior chamber, vitreous or orbit. Eosinophilia may occur. CT scan and MRI shows intracranial and ocular cysts. Ocular ultrasonography helps to locate intraorbital and intraocular coenurus. The dead larvae cause uveitis.

There is no specific treatment. Praziquantel 50 $\mathrm{mg} / \mathrm{kg} /$ day in divided doses for fifteen days may be administered. However, both definitive diagnosis and treatment require surgical excision of the lesion.

\section{Trematodes (Flukes) \\ Schistosoma Species}

The parasites involved are various species of Schistosoma (S. haematobium, S. japonicum and S. mekongi). The worms have a complicated life cycle. Sexually mature worms live in pairs in veins. Ova are secreted in the urogenital system or the gut. The miracidia hatch in fresh water and infect the intermediate host (snail). Asexual multiplication occurs in the snail and cercaria larva are released in the water. These cercaria actively burrow through the skin of the definitive host (man) or may burrow through the mucous membrane if ingested orally. After 2 to 3 months, mature worms develop within the venous system. S. haematobium may have other definitive hosts like dogs, cats, pigs and cattle, but the other Schistosomas infect man only.

The disease is prevalent in the hot, humid climates of Africa, Brazil and East Asia. The flukes cause granulomas in the eyes, particularly in the conjunctiva, lacrimal gland, choroid and retina. The disease is diagnosed after histological examination of the excised biopsy specimens and by ELISA.

The treatment involves excision of the granulomas. Praziquantel $40 \mathrm{mg} / \mathrm{kg} /$ day is given on one day in two separate doses for S. haematobium and S. mansoni. A higher dose of $60 \mathrm{mg} / \mathrm{kg} /$ day is to be used for $\mathrm{S}$. japonicum and $\mathrm{S}$. mekongi. This is followed by niridazole $12.5 \mathrm{mg} / \mathrm{kg}$ twice daily for 7 days. Other options are oxamniquine, metrifonate and amoscanate.

\section{Paragonimus westermani}

This causes paragonimiasis. It is a lung fluke. The parasite lives in human lung. The eggs reach the mouth of the bronchi and are then swallowed and excreted through the faeces. The cercaria again infect the snails and then the crabs. Human beings get infected by eating the undercooked crabs. The metacercaria excyst in the small gut, penetrate its wall, migrate through the diaphragm into the lungs. Here they grow into adults. Adult worms can live upto ten years. The disease occurs in India, East Asia, Africa and also in Central and South America.

The eyes are usually affected in the form of recurrent severe uveitis or secondarily due to DUSN. ${ }^{74}$ The diagnosis is made by the highly specific ELISA test.

Surgical removal of the parasite is the treatment of choice. Medical therapy is ineffective for intraocular parasites.

\section{Diseases caused by Arthropods Pediculosis and Phthiriasis}

Infestation with the crab louse, Pthirus pubis, is commonly associated with eyelashes (cilia). The mode of transmission is sexual. Pediculosis is head and body louse infestation.

Blepharitis and haemorrhagic crusts occur on the lid margins. The insects can be seen as well as their faeces (scaly brown in colour, amongst the cilia) and louse eggs (nits).

The treatment consists of manual removal by forceps and epilation of affected cilia 1\% silver nitrate may be applied followed by corticosteroid ointment. The ointment is not ovicidal, so it has to be repeated several times for two weeks to kill the newly hatched lice $1 \%$ yellow mercuric oxide ointment also is to be used for two weeks. ${ }^{75}$ Lice may also be killed with $20 \%$ fluorescein solution. ${ }^{76}$ Cryotherapy kills both lice and their nits. ${ }^{77}$

\section{Tick Disease}

The ticks transmit various diseases such as encephalitis, Borreliosis (Lyme disease), Rocky Mountain, Spotted River and $\mathrm{Q}$ fever. Infection with one species may prevent infection with other rickettsial species subsequently. ${ }^{78}$ The tick must be removed intact with minimal manipulation by the use of fine, flat, ophthalmic forceps. They cause conjunctival congestion, subconjunctival haemorrhage, retinal haemorrhage and nerve palsies. Treatment comprises treatment of the systemic disease. 


\section{Ophthalmomyiasis}

This is an ocular disease caused by infestation of larvae of flies. This is a rare disease, which mainly occurs in the tropics and subtropics. It is also seen in the Balkan countries.

The flies lay their eggs (Ovipara) or live larvae (Vivipara) on a substrate, on which the larvae feed and grow before encapsulation into a cocoon. Thereafter, it undergoes metamorphosis into the adult flying stage. The larvae may also leave the substrate and drop to the ground and burrow into the soil or hide in other places.

Several places may serve as the substrate for the maggot, e.g. gut, urogenital tract, nasal cavities and the eye and its adnexa. The larvae can invade the living tissue or may remain on the surface. The larvae may be directly deposited or may be deposited via a stinger. Once they are deposited they cling to surface tissues by means of hooks. They can then penetrate deeper into the tissues.

Ophthalmomyiasis may be external or internal. External ophthalmomyiasis is a conjunctivitis caused by fly larvae (maggots). The flies involved are Oestrus ovis and Rhinoestrus purpureus. There is a foreign body sensation. Conjunctiva becomes congested and chemosed. Slit lamp examination shows maggots clinging to the conjunctiva. They are mobile and exhibit negative phototaxis. The prognosis is good, as there is no tissue invasion. ${ }^{79}$

The treatment includes manual removal under topical anaesthesia with $5 \%$ to $10 \%$ cocaine. ${ }^{79}$ Other agents which may be used include cholinesterase inhibitors. ${ }^{80}$

Internal ophthalmomyiasis occurs when the larvae invade the globe. Endophthalmitis may occur especially in children presenting with a softer sclera. ${ }^{79}$ The involved species are Hypoderma bovis, W. magnifica and Oestrus ovis. The larvae burrow through the conjunctiva and reach the limbs. From the limbus, they may enter the anterior chamber (anterior internal ophthalmomyiasis) and when they invade the posterior segment, the condition is called posterior internal ophthalmomyiasis.

The treatment is mainly surgical, but laser photocoagulation may be used. If the larva is yet to enter the posterior segment, its surgical removal may save the eye. The larva may be removed from the sclera or the anterior chamber. If the larva has migrated to the posterior segment, the prognosis is much worse. Photocoagulation and vitrectomy may be tried. In case the inflammation is minimal, no medical therapy may be needed.

Ophthalmomyiasis furunculosis (also called unilarval myiasis) is a clinical situation where there is infestation by Dermatobia hominis. There is infestation by a single larva which initially manifests as an abscess, which opens at the surface through which the single larva protrudes out to take oxygen. The treatment involves removal with forceps after blocking the respiratory pore with ointment. Local anaesthesia may be required, especially if the burrow is to be incised to facilitate worm removal.

Recurrent exophthalmos may be caused by Hypoderma bovis maggots, which penetrate orbital tissues. There is oedema of the eyelids and face. There is eosinophilia. Palliative therapy may be done with antibiotics and corticosteroids. Surgical removal of the maggots may be done. Infested tissue space may be irrigated and gauze packed with formalin, turpentine or carbolic acid. If all treatment fails, exenteration may be tried as a last resort.
Destructive ophthalmomyiasis occurs when carnivorous tropical maggots attack necrotic malignant tissues with a necrotising ulcer. The orbital structures are severely damaged including the eyeball and the orbital walls. Meningitis can occur as a complication. The treatment includes topical anaesthesia with $10 \%$ cocaine and then manual removal. The ulcer must be cleaned with $0.1 \%$ potassium permanganate solution or $1 \%$ silver nitrate solution. Necrotic tissues may be debrided. Systemic and topical antibiotic therapy is given.

\section{Mechanical Transfer of Disease by Arthropods}

In this situation, the vectors involved are not a part of the life cycle of the parasite. Domestic flies can mechanically transmit Chlamydia trachomatis (causing trachoma) and bacteria causing infective conjunctivitis.

Among the fly control measures, environmental measures are the most important. ${ }^{81}$ Storage of garbage and kitchen wastes in closed containers, proper disposal of human and animal excreta and proper refuse disposal- all are important. Insecticides, larvicides, fly baits, space sprays and fly paper may also be used in conjunction with environmental measures. Wire mesh screen in household windows and wire mesh cover for foodstuffs may prevent access of flies to foodstuffs.

\section{Scopes of Telepathology in the Diagnosis of Ocular Parasites}

This process involves the use of telecommunication technology at remote locations by viewing images on a visual display unit (computer) instead of direct visualisation in the bright field light microscope. The collection and preparation of the samples are done by trained medical laboratory technicians posted at remote locations. They feed the images to the computer for on-line transmission. On an average, each case comprises of 5 images (range 4 - 6) with a total dimension of 300 kilobytes. The mean time needed for acquisition of imaging in each case is on an average 3 minutes and an average of 5 minutes for transmission resulting in an overall time of 8 minutes. The teleophthalmic pathology consultant views the images and gives the report of the diagnosis. If necessary, the teleopthalmic pathology consultant may view the slide directly before giving the report. 82

\section{CONCLUSION}

The parasites are responsible for some of the devastating diseases around the globe, both in human beings as well as animals.1,2 Eye damage can occur directly by the parasite or indirectly due to toxic products, immune responses or ectopic parasitism by the pre-adult or adult stages of the parasite.

It is important to review each patient and this should be done by both ophthalmologists and microbiologists. Removal of the live worm from the eye is important, as the parasitic body fluids are highly toxic to the intraocular structures. Severe intraocular inflammation and infection and even endophthalmitis have been known to occur.1,2

Thus, a proper diagnosis can save the eyes and the life of a patient by instituting appropriate treatment at the appropriate time. The ocular microbiologist can give useful information by following appropriate algorithms, ${ }^{83}$ to the ophthalmologist, about the diagnosis of the ocular parasite in 
the laboratory. This would facilitate better management of the patient, not only in the cities, but also in remote areas with the use of teleophthalmic pathology facilities in the near future. ${ }^{82}$

\section{REFERENCES}

[1] Nimir AR, Saliem A, Ibrahim IA. Ophthalmic parasitosis: a review article. Interdiscip Perspect Infect Dis 2012;2012:587402.

[2] Rathinam SR, Annamalai R, Biswas J. Intraocular parasitic infections. Ocul Immunol Inflamm 2011;19(5):327-36.

[3] Peak E, Hoffmann KF. Cross-disciplinary approaches for measuring parasitic helminth viability and phenotype. An Acad Bras Ciene 2011;83(2):649-62.

[4] Wilhelmus KR, Osato MS, Font RL, et al. Rapid diagnosis of Acanthamoeba keratitis using calcofluor white. Arch Ophthalmol 1986;104(9):1309-12.

[5] Lehmann OJ, Green SM, Morlet N, et al. Polymerase chain reaction analysis of corneal epithelial and tear samples in the diagnosis of Acanthamoeba keratits. Invest Ophthalmol Vis Sci 1998;39(7):1261-5.

[6] Lakshmi SG, Biswas J, Natarajan K, et al. Acanthamoeba keratitis - clinicopathological study of 6 cases. Afro-Asia J Ophthalmol 1993;11(1):9-18.

[7] Henderly DE, Genstler AJ, Smith RE, et al. Changing patterns of uveitis. Am J Ophthalmol 1987;103(2):131-6.

[8] McCannel CA, Holland GN, Helm CJ, et al. Causes of uveitis in the general practice of ophthalmology. UCLA Community Based Uveitis Study Group. Am J Ophthalmol 1996;121(1):35-46.

[9] Nijhawan R, Bansal R, Gupta N, et al. Intraocular cysts of Toxoplasma gondii in patients of necrotizing retinitis following periocular/intraocular triamcinolone injection. Ocul Immunol Inflamm 2013;21(5):396-9.

[10] Borkowshi PK. New trends in ocular toxoplasmosis the review. Przegl Epidemiol 2001;55(4):483-93.

[11] Da Mata AP, Orefice F. Toxoplasmosis. In: Lampert R, di Berardino C. eds. Diagnosis and treatment of uveitis. Philadelphia: WB Saunders Company., 2002:385-99.

[12] Freitas-Neto CA, Cao JH, Orefice JL, et al. Increased submacular choroidal thickness in active, isolated extramacular toxoplasmosis. Ophthalmology 2016;123(1):222-4. e1.

[13] Diesenhouse MC, Wilson LA, Corrent GF, et al. Treatment of microsporidial keratoconjunctivitis with topical fumagillin. Am J Ophthalmol 1993;115(3):2938.

[14] Edman JC, Kovacks JA, Masur H. Ribosomal RNA sequence shows Pneumocystis carinii to be a member of the fungi. Nature 1998;334:519-22.

[15] Telzak EE, Cote RJ, Gold JWM, et al. Extrapulmonary Pneumocystis carinii infections. Rev Infect Dis 1990;12(3):380-6.

[16] Salmon-Ceron D, Fontbonne A, Saba J, et al. Lower survival of AIDS patients receiving dapsone compared with aerosolised pentamidine for secondary prophylaxis of Pneumocystis carinii pneumonia. Study Group. J Infect Dis 1995;172(3):656-64.
[17] Veraldi S, Bottini S, Curro N, et al. Leismaniasis of the eyelid mimicking an infundibular cyst and review of the literature on ocular leishmaniasis. Int J Infect Dis 2010;14(Suppl 3):e230-2.

[18] Conture S, Agrawal R, Woods K, et al. A case of panuveitis with hypopyon due to presumed ocular leishmaniasis in a HIV patient. J Ophthalmic Inflamm Infect 2014;4(1):21.

[19] Zadeh MM, Manshai K, Shaddel M, et al. Ocular leishmaniasis. Iran J Ophthalmol 2006;19(3):1-5.

[20] Biswas J, Mani B, Bhende M. Spontaneous resolution of bilateral macular haemorrhage in a patient with Kala-azar. Eye (Lond) 2000;14(Pt 2):244-6.

[21] Hidayat AA, Nalbandian RM, Sammons DW, et al. The diagnostic histopathologic features of ocular malaria. Ophthalmology 1993;100(8):1183-6.

[22] Biswas J, Fogla R, Srinivasan N, et al. Clinical and histopathological findings of ocular malaria - a study of three cases. Ophthalmology 1996;103:9.

[23] Biswas J, Fogla R, Srinivasan P, et al. Ocular malaria. A clinical and histopathologic study. Ophthalmology 1996;103(9):1471-5.

[24] Beare NA, Taylor TE, Harding SP, et al. Malarial retinopathy: a newly established diagnostic sign in severe malaria. Am J Trop Med Hyg 2006;75(5):790-7.

[25] Carroll ME, Anast BP, Birch CL. Giardiasis and uveitis. Arch Ophthalmol 1961;65(6):775-8.

[26] Dent JH, Nichols RL, Beaver PC, et al. Visceral larva migrans: with a case report. Am J Pathol 1956;32(4): 777-803.

[27] Rangel TR, Foster CS. Ocular toxocariasis. In: Lampert, Di Berardino C. eds. Diagnosis and treatment of uveitis. Philadelphia: WB Saunders Company 2002:428-36.

[28] Jee D, Kim KS, Lee WK, et al. Clinical features of ocular toxocariasis in adult Korean patients. Ocul Immunol Inflamm 2016;24(2):207-16.

[29] Mahesh G, Giridhar A, Biswas J, et al. A case of periocular dirofilariasis masquerading as a lid tumour. Indian J Ophthalmol 2005;53(1):63-4.

[30] Mittal M, Sathish KR, Bhatia PG, et al. Ocular dirofilariasis in Dubai, UAE. Indian J Ophthalmol 2008;56(4):325-6.

[31] Logar J, Novsak V, Rakovec S, et al. Subcutaneous infection caused by Dirofilaria repens imported to Slovenia. J Infect 2001;42(1):72-4.

[32] Das D, Das K, Islam S, et al. A rare case of anterior chamber dirofilariasis. Oman J Ophthalmol 2015;8(1):50-3.

[33] Pauly M, Biswas J, Hussain RN, et al. Periocular dirofilariasis mimicking lacrimal sac mucocoele. Orbit 2013;32(5):324-6.

[34] Agarwal M, Biswas J. Live Intraocular dirofilaria causing multifocal chorioditis. Retin Cases Brief Rep 2009;3(2):228-9.

[35] Gorezis S, Psilla M, Asprondis I, et al. Intravitreal dirofilariasis: a rare ocular infection. Orbit 2006;25(1):57-9.

[36] Karyakarte R, Damle A. Medical parasitology. $3^{\text {rd }}$ edn. Kolkata: Books \& Allied (P) Ltd., 2012:210-11.

[37] Karyakarte R, Damle A. Medical parasitology. $3^{\text {rd }}$ edn. Kolkata: Booked \& Allied (P) Ltd., 2012:188-9. 
[38] Kean BH, Sun T, Ellsworth RM. Colour Atlas / Text of ophthalmic parasitology. New York: Igaku-Shoin 1991.

[39] Frierson JG, Trichinosis. In: Goldsmith R, Heynemann D. eds. Tropical medicine and parasitology. Norwalk, CT: Appleton \& Lange 1989: p. 423.

[40] Goldberg MA, Kazakos KR, Boyce WM et al. Diffuse unilateral subacute neuroretinitis. Morphometric, serologic and epidemiologic support for Baylisascaris as a causative agent. Ophthalmology 1993;100(11): 1695-701.

[41] Kuchle M, Knorr HL, Medenblik-Frysch S, et al. Diffuse unilateral subacute neuroretinitis syndrome in a German most likely caused by the raccoon roundworm Baylisascaris procyonis. Graefe's Arch Clin Exp Ophthalmol 1993;231(1):48-51.

[42] Gass JD, Callanan DG, Bowman CB. Oral therapy in diffuse unilateral subacute neuroretinitis. Arch Ophthalmol 1992;110(5):675-80.

[43] Ho Yuen V, Chang TS, Hooper PL. Diffuse unilateral subacute neuroretinitis syndrome in Canada. Arch Ophthalmol 1996;114(10):1279-82.

[44] Rodger FC. The pathogenesis and pathology of ocular onchocerciasis. Am J Ophthalmol 1960;49:104-9.

[45] WHO Expert Committee. Report of a WHO Expert Committee on Onchocerciasis Control. Technical Report Series No: 752. Geneva: WHO, 1987.

[46] Chan CC, Li Q, Brezin AP, et al. Immunopathology of ocular oncherciasis 3. Th-2 helper $\mathrm{T}$ cells in the conjunctiva. Ocul Immunol Inflamm 1993;1(1-2):71-8.

[47] Van der Lelij A, Doekes G, Hwan BS, et al. Humoral autoimmune response against $\mathrm{S}$-antigen and IRBP in ocular onchocerciasis. Invest Ophthalmol Vis Sci 1990;31(7):1374-80.

[48] Barua P, Sharma A, Hazarika NK, et al. A rare case of ocular onchocerciasis in India. South East Asian J Trop Med Public Health 2011;42(6):1359-64.

[49] Das D, Islam S, Bhattacharjee $\mathrm{H}$, et al. Parasitic diseases of zoonotic importance in humans of northeast India, with special reference to ocular involvement. Eye Brain 2014;6:1-8.

[50] Bhattacharjee H, Das D, Medhi J. Intravitreal gnathostomiasis and review of literature. Retina 2007;27(1):67-73.

[51] Basak SK, Sinha TK, Bhattacharya D, et al. Intravitreal live Gnathostoma Spinigerum. India J Ophthalmol 2004;52(1):57-8.

[52] Biswas J, Gopal L, Sharma T, et al. Intraocular Gnathostoma spinigerum. Clinicopathologic study of two cases with review of literature. Retina 1994;14(5):438-44.

[53] Maleewong W, Sithitaworn P, Tesana S, et al. Scanning electron microscopy of the early third stage larvae of Gnathostoma spinigerum. Southeast Asian J Trope Med Public Health 1988;19(4):643-7.

[54] Anantaphruti M, Setasuban P, Daengsvang S, et al. Electron microscopy of the advanced third stage larvae of Gnathostoma spinigerum. Southeast Asian J Trop Med Public Health 1982;13(4):531-40.
[55] Sangwan VS. Ascariasis. In: Lampert R, Di Berardino C. eds. Diagnosis and treatment of uveitis. Philadelphia, USA: WB Saunders Company 2002:437-42.

[56] Balasubramaniam M, Sudhakar P, Subhashini M, et al. Ascaris lumbricoides in the lacrimal passage. Indian J Ophthalmol 2000;48(1):53-4.

[57] Roy FH. Ocular syndromes and systemic diseases. $5^{\text {th }}$ edn. New Delhi: Jaypee Brothers 2014.

[58] Elsdondew R, Freedman L, Kaplan CS. A worm in the eye: a familiar parasite in an unusual situation. S Afr Med J 1956;30(33):791-2.

[59] El-Shabrawi Y. Loiasis. In: Lampert R, Di Berardino C, eds. Diagnosis and treatment of uveitis. Philadelphia: WB Saunders Company 2002:463-7.

[60] Tyagi P, Arsenio M, Bekir OA, et al. Subconjunctival Loa loa. BMJ Case Rep 2011:2011. Pii Bcr 0620103075.

[61] Osuntokum 0, Olurin O. Filarial worm (Loa loa) in the anterior chamber. Report of two cases. Br J Ophthalmol 1975;59(3):166-7.

[62] Toussaint D, Danis P. Retinopathy in generalised Loa loa filariasis. A clinicopathological study. Arch Ophthalmol 1965;74(4):470-6.

[63] Karyakarte R, Damle A. Medical parasitology. $3^{\text {rd }}$ edn. Kolkata: Books and Allied (P) Ltd., 2012: p. 191.

[64] Ganesh SK, Babu K, Krishnakumar S, et al. Ocular filariasis due to Wuchereria bancrofti presenting as panuveitis: a case report. Ocul Immunol Inflamm 2003;11(2):145-8.

[65] Karyakarte R, Damle A. Medical parasitology. $3^{\text {rd }}$ edn. Kolkata: Books and Allied (P) Ltd., 2012:197-203.

[66] Sharma $\mathrm{T}$, Sinha $\mathrm{S}$, Shah $\mathrm{N}$, et al. Intraocular cysticercosis: clinical characteristics and visual outcome after vitrectomy surgery. Ophthalmology 2003;110(5):996-1004.

[67] Mahendradas P, Biswas J, Khetan V. Fibrinous anterior uveitis due to cysticercus cellulosae. Ocul Immnol Inflamm 2007;15(6):451-4.

[68] Sihota R, Honavar SG. Oral albendazole in the management of extraocular cysticercosis. $\mathrm{Br} J$ Ophthalmol 1994;78(8):621-3.

[69] Yanoff M, Sassani JW. Ocular pathology. $6^{\text {th }}$ edn. Philadelphia: Elsevier 2009: p. 92.

[70] Mahesh L, Biswas J, Subramanian N. Role of ultrasound and CT Scan in diagnosis of hydatid cyst of the orbit. Orbit 2000;19(3):179-88.

[71] Mukherjee B, Biswas J, Raman M. Subconjunctival larva migrans caused by sparganum. Indian J Ophthalmol 2007;55(3):242-3.

[72] Karyakarte R, Damle A. Medical parasitology. $3^{\text {rd }}$ edn. Kolkata: Books \& Allied (P) Ltd., 2012: p. 147.

[73] Karyakarte R, Damle A. Medical parasitology. 3rd edn. Kolkata: Books \& Allied (P) Ltd., 2012: p. 144.

[74] Gass JD, Braunstein RA. Further observations concerning the diffuse unilateral subacute neuroretinitis syndrome. Arch Ophthalmol 1983;101(11):1689-97.

[75] Ashkenazi J, Desatnik HR, Abraham FA. Yellow mercuric oxide: a treatment of choice for phthiriasis palpebrarum. Br J Ophthalmol 1991;75(6):356-8. 
[76] Mathew M, D'Souza P, Mehta DK. A new treatment of phthiriasis palpebrarum. Ann Ophthalmol 1982;14(5):439-41.

[77] Awan KJ. Cryotherapy in phthiriasis palpebrarum. Am J Ophthalmol 1997;83(6):906-7.

[78] Weiss E. The biology of rickettsial diseases. Annu Rev Microbiol 1982;36:345.

[79] Behr C. Uber ophthalmomyiasis interna und externa. Die Fliegenlarvener Krangkung des Auges. Klin monatsbi Augenheikel 1920;64:161-80.

[80] Linnen HJ. Ophthalmomyiasis externa, irhe moglichen Folgeerkranskungen und Therapie. Klin Monatsbi Augenheikel 1951;119:19-23.
[81] Karyakarte R, Damle A. Medical parasitology. $3^{\text {rd }}$ edn. Kolkata: Books \& Allied (P) Ltd., 2012: p. 230.

[82] Biswas J, Krishnakumar S, Ahuja S. Manual of ocular pathology. New Delhi: Jaypee-Highlights Medical Publishers Inc., 2010: p. 118.

[83] Das D, Ramachandra V, Islam S, et al. Update on pathology of ocular parasitic disease. Indian J Ophthalmol 2016;64(11):794-802. 